\title{
Article
}

\section{Oil film separation and drag torque in disengaged wet brakes}

Morris, Nick, Davies, James A., Leighton, Michael, King, Paul and Rahnejat, Homer

Available at http://clok.uclan.ac.uk/32562/

Morris, Nick, Davies, James A., Leighton, Michael, King, Paul and Rahnejat, Homer ORCID: 0000-0003-2257-7102 (2020) Oil film separation and drag torque in disengaged wet brakes. Proceedings of the Institution of Mechanical Engineers, Part D: Journal of Automobile Engineering, 234 (1). pp. 17-27. ISSN $0954-4070$

It is advisable to refer to the publisher's version if you intend to cite from the work. http://dx.doi.org/10.1177/0954407019844358

For more information about UCLan's research in this area go to http://www.uclan.ac.uk/researchgroups/ and search for < name of research Group $>$.

For information about Research generally at UCLan please go to http://www.uclan.ac.uk/research/

All outputs in CLoK are protected by Intellectual Property Rights law, including Copyright law. Copyright, IPR and Moral Rights for the works on this site are retained by the individual authors and/or other copyright owners. Terms and conditions for use of this material are defined in the policies page.

\section{CLoK}

Central Lancashire online Knowledge www.clok.uclan.ac.uk

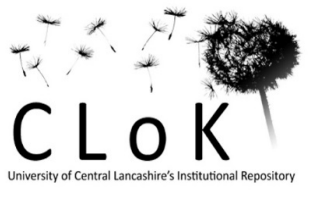


This item was submitted to Loughborough's Research Repository by the author.

Items in Figshare are protected by copyright, with all rights reserved, unless otherwise indicated.

\section{Oil film separation and drag torque in disengaged wet brakes}

PLEASE CITE THE PUBLISHED VERSION

https://doi.org/10.1177/0954407019844358

PUBLISHER

SAGE Publications

VERSION

AM (Accepted Manuscript)

\section{PUBLISHER STATEMENT}

This work is made available according to the conditions of the Creative Commons Attribution-NonCommercialNoDerivatives 4.0 International (CC BY-NC-ND 4.0) licence. Full details of this licence are available at: https://creativecommons.org/licenses/by-nc-nd/4.0/

\section{LICENCE}

CC BY-NC-ND 4.0

\section{REPOSITORY RECORD}

Morris, Nick, James A. Davies, Michael Leighton, Paul King, and Homer Rahnejat. 2019. "Oil Film Separation and Drag Torque in Disengaged Wet Brakes". figshare. https://hdl.handle.net/2134/37457. 


\title{
Oil Film Separation and Drag Torque in Disengaged Wet Brakes
}

N. Morris*, J. Davies, M. Leighton, P. D. King, H. Rahnejat

Loughborough University, Loughborough, UK

*Corresponding author

\begin{abstract}
Multi-disc wet brake packs are commonly used for off-highway applications. Unfortunately when disengaged, these components can create significant levels of unwanted parasitic losses. This paper presents a novel, validated model to predict the parasitic losses in disengaged multi-disc wet brake packs. The model employs pertinent boundary conditions to determine the position of the free boundary created by oil film separation. The experimental and analytical results demonstrate the significance of wet brake disengaged losses at a range of angular velocities, typically found in operation. The improved understanding accrued by the expounded time-efficient methodology presents significant opportunity to develop predictive tools for driveline system efficiency calculations and component design optimisation for off-highway vehicles.
\end{abstract}

Keywords: Wet Brake system, Wet Clutch, Lubricant Drag, friction, Off-highway vehicles

\section{Nomenclature}

\begin{tabular}{ll}
$c_{p}$ & Specific heat capacity \\
$\mathrm{C}$ & Integration constant \\
$\mathrm{D}$ & Integration constant \\
$h_{g}$ & Stator- brake disc axial separation \\
$h_{p}$ & Stator- brake disc groove axial separation \\
$n$ & Stator-brake disc pad axial separation \\
$p$ & number of brake disc grooves \\
$p_{i}$ & Mean pressure across oil film \\
$P e$ & Pressure at the inner radius \\
$p_{o}$ & Dimensionless Peclet number \\
$P$ & Pressure at the free boundary \\
$Q$ & Power loss \\
$r$ & Oil flow rate in the radial direction \\
\hline
\end{tabular}




\begin{tabular}{ll}
$R_{e}$ & Brake disc external radius \\
$R_{i}$ & Brake disc inner radius \\
$R_{o}$ & Free boundary radial position \\
$w$ & Brake disc groove width \\
$T$ & Drag torque \\
$u$ & Radial velocity \\
$v$ & Tangential velocity \\
$w$ & Brake disc groove width \\
$z$ & Position in axial direction \\
Greek letters & \\
$\theta$ & Lubricant temperature \\
$\theta_{0}$ & Bulk Lubricant temperature \\
$\rho$ & Density \\
$\eta$ & Effective dynamic viscosity \\
$\eta_{0}$ & Dynamic viscosity at reference temperature \\
$\lambda$ & Thermal conductivity of oil \\
\hline &
\end{tabular}

\section{Introduction}

Hydraulically-actuated multi-disc wet brake pack systems constitute the system of choice for many off-highway vehicles $[1,2]$. The wet brake packs are commonly situated between the differential and final drive epicyclic gear set which is located in the wheel hub assembly. The brake packs typically comprise a series of friction discs and separators (stators) which are lugged to the brake pack housing. The brake discs have a carbon or paper-based lining and are splined to the axle shaft. The brake discs are typically immersed close to their centre-line in a sump of suitable lubricant.

In a similar manner to wet clutches, disengaged wet brake packs can experience significant losses due to viscous drag [3-6]. Leighton et al [6] presented a combined experimental-numerical study of partially submerged, centrifugally-fed wet brake losses at different sump temperatures. It was shown that the combination of cold start-up and high-speed road driving conditions would lead to a $20 \mathrm{Kw}$ power loss. It was also shown that a coherent film of lubricant could not be maintained between the discs under some operating conditions. Similar findings have been reported for wet clutches with a lubricant supply through a central shaft [7-9]. The breakdown of a coherent film of lubricant is 
generally attributed to onset of cavitation $[6,10]$. The presumption that cavitation is the dominant mechanism in oil film rupture means that analytical solutions for disengaged wet clutch drag torque usually employ Swift [11]-Stieber[12] (also referred to as Reynolds') boundary conditions.

Observation of lubricant film rupture in rotary components, separated by a thin lubricant film shows a single free boundary is often formed, with the remaining volume of lubricant swept away by the rotating surface. Examples of this are reported for wet clutches $[8,13,14]$, as well as for rotary face seals $[15,16]$. This indicates that wet brakes and clutch packs should use a separation (PrandtlHopkins incipient reverse flow) boundary conditions rather than the commonly employed cavitation boundary conditions. These boundary conditions have been applied in the case of rolling and sliding contacts of gears and bearings and verified successfully through combined numerical-experimental studies $[17,18]$. The separation boundary conditions have also been verified for the sliding contact of piston compression ring to cylinder liner in detailed computational thermo-fluid dynamics with the inclusion of vapour transport equation [19].

This paper presents a new thermo-hydrodynamic model with an implicit formulation to predict partially submerged, centrifugally-fed disengaged wet brake losses. The model uses an appropriate separation (incipient reverse flow) boundary condition, rather than the commonly employed cavitation boundary condition. Another advantage of the model is in its non-reliance on the determination of lubricant supply flow rate. Instead, it merely relies on the known pressures at the internal and outer disc radii. The model is validated using a test rig operating at various angular velocities and sump temperatures. The time-efficient model enables accurate component design and prediction of driveline system efficiency such as that presented by Jenane et al [3].

\section{Experimental Methodology}

Commonly, lubricant is supplied to wet brake packs through their partial immersion into a sump (4) and via centrifugal splashing (see figure 1). This is preferred rather than the pressurised oil feed method, which is more commonly used for wet clutch applications. A test rig is devised to represent the disengaged brake configuration for a single brake friction disc and a stator pair as shown in figure 1. The front panel of the sump (4) is hidden in figure 1 so that the working mechanism can be clearly observed. The primary purpose of the rig is to measure the frictional drag losses under different operating conditions; temperature and sliding speed. A detail description of the test rig is also provided in Leighton et al [6]. The rig comprises an electric motor (1), connected to an inline torque limiting coupling (2), rotatory torque transducer (3) and an immersed brake disc (5) that rotates between the two stator discs (6). The separation is set using gauge blocks placed between the two stator discs (7). This enables the brake disc to axially align to the stator pair.

A rigorous test procedure is pursued to remove the influence of any ancillary component on the measurements. The test rig is run under dry sump conditions for 10 minutes in order to allow the support bearings to reach the stable operating temperature. Then, a baseline measurement is taken in order to determine the contribution to the loss of torque due to the ancillary components. Subsequently, these measured losses are subtracted from the measurements taken with the sump (4) filled to the shaft centre line (representative of wet brake operating conditions). The sump (4) temperature is altered by a heating element (6), which is used in conjunction with a control unit and thermocouple to regulate the sump temperature. 


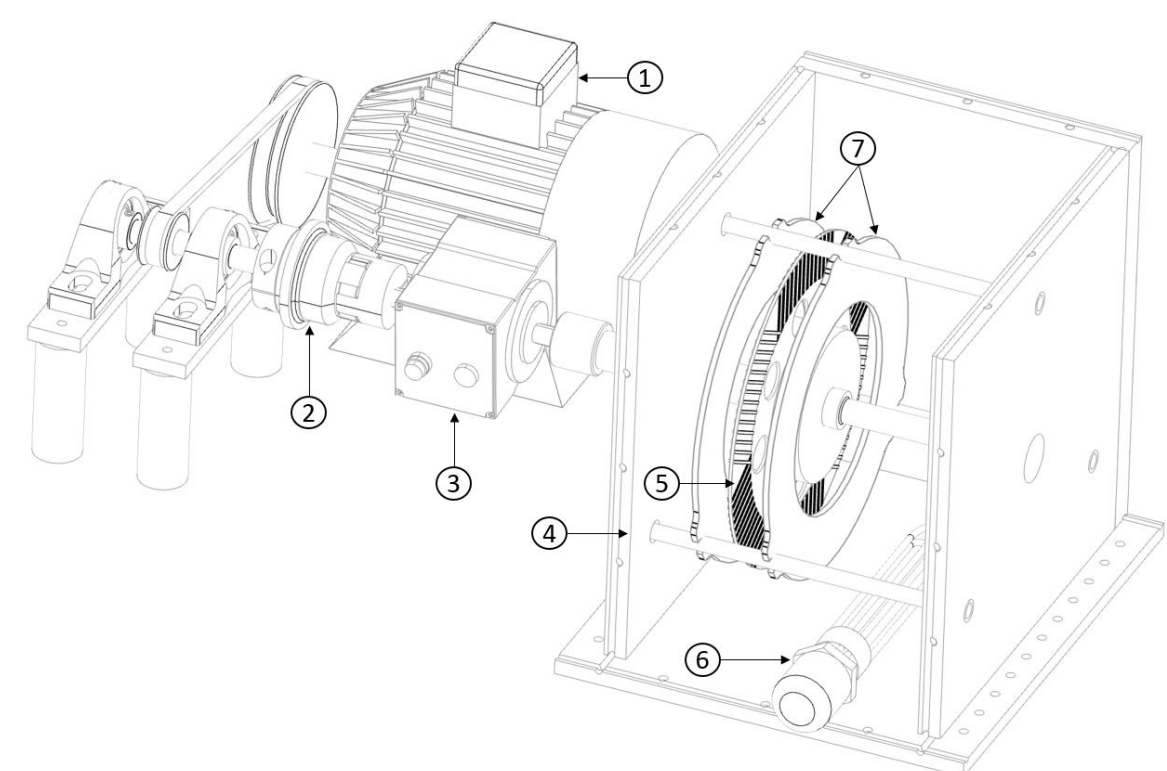

Figure 1: Disengaged wet brake test rig (with the sump front plate hidden)

\section{Thermo-Hydrodynamic model}

The model herewith presented considers the flow of lubricant between the annular brake and stator plates using a cylindrical coordinate system. The fluid flow in the radial direction including centrifugal and Poiseuille flow is used to determine the position of film rupture at which the free boundary is formed (in fluid dynamics a free boundary is considered to be a boundary at a constant pressure [20]). In the tangential direction the Couette flow is considered to determine the parasitic drag losses generated by the fluid film formed between the inner radius and the free boundary. Figure 2 shows the radial flow through a cross-section of a single brake stator conjunction. 


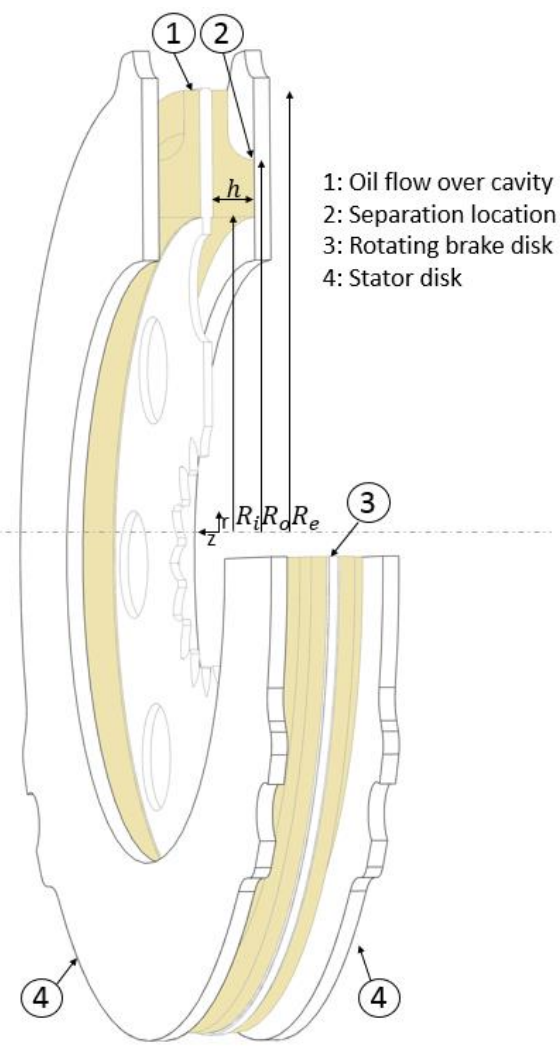

Figure 2: Representation of Wet brake pack contact model

The dimensions $R_{i}, R_{o}$ and $R_{e}$ are the inner radius of the brake disc (rotating disc), oil film separation position and the external radius of the brake disc (rotating disc) respectively. The axial gap between the plates is described by the term $h$. Dowson [23] showed that the radial velocity can be determined as a function of cross-film position using a simplified form of Navier-Stokes equation in cylindrical coordinates. It is assumed that the conjunction is axisymmetric under steady state conditions, and that the body forces may be neglected. When the lubricant can be considered incompressible and isoviscous the radial velocity can be determined as a function of cross-film position as:

$u(z)=-\frac{z(h-z)}{2 \eta} \frac{d p}{d r}+\frac{r \rho \omega^{2} z\left(h^{3}-z^{3}\right)}{12 \eta h^{2}}$

While the continuity of flow can be described as:

$\int_{0}^{h} \frac{1}{r} \frac{\partial u r}{d r} d z=0$

Dowson [23] showed that the following second order differential equation (3) can be obtained by the substitution of equation (1) into the continuity equation (2), hence:

$\frac{d}{d r}\left(r \frac{d p}{d r}\right)=\frac{3}{5} \rho r \omega^{2}$

Integration of equation (3) with respect to $r$ leads to a relationship between the fluid pressure and radial position (figure 2 ) as:

$\frac{d p}{d r}=\frac{3}{10} \rho r \omega^{2}+\frac{C}{r}$ 
$p=\frac{3}{20} \rho r^{2} \omega^{2}+C \ln r+D$

Clearly, three equations are needed to determine the unknown integration constants $C$ and $D$ and the location of the free boundary $R_{O}$ (figure 2). Two equations (7) and (8) can be written by applying the pressures at the inner $R_{i}$ and outer radius $R_{o}$ shown in equation (6) and equation (5).

$p=p_{i}$ at $r=R_{i}$ and $p=p_{o}$ at $r=R_{o}$

$D=p_{i}-\frac{3}{20} \rho R_{i}^{2} \omega^{2}-C \ln R_{i}$

$D=p_{o}-\frac{3}{20} \rho R_{o}^{2} \omega^{2}-C \ln R_{o}$

The third equation required to determine the three unknown can be found by determining the pressure gradient required for incipient counter flow (separation) boundary [20-22]. The free boundary caused by fluid film Separation is characterised by determining the location of incipient zero reverse flow (Prandtl-Hopkins condition) which can be written as:

$\frac{d u}{d z}=u=0$

For the current system this condition will occur at the stationary surface where $z=0$. Therefore, by combining equations (1) and (9) the pressure gradient in the radial direction at which the fluid film separation occurs can be written as:

$\frac{d p}{d r}=\frac{r \rho \omega^{2}}{6}$

Equations (10) and (4) equate at: $r=R_{O}$. Therefore, the first integration constant can be written as:

$C=-\frac{2 \rho R_{0}^{2} \omega^{2}}{15}$

By combining equations (7), (8) and (11), equation (12) can be written with the dimensionless Euler number on the left-hand side and a geometric relation on its right-hand side:

$\frac{p_{i}-p_{o}}{\rho R_{o}^{2} \omega^{2}}=\frac{3}{20}\left(\frac{R_{i}{ }^{2}}{R_{o}^{2}}-1\right)-\frac{2}{15} \ln \left(\frac{R_{i}}{R_{o}}\right)$

The implicit formulation shown in equation (12) can then be solved iteratively to find the value of $R_{O}$ to satisfy the equation, where $R_{o}$ varies between the internal radius $R_{i}$ and the external disc radius $R_{e}$ when $p_{i}-p_{o}<0$.Such conditions have been shown to be plausible by experimental measurements carried out by Pahlovy et al [9]. These are shown be reasonable when the lubricant flow rate is less than the centrifugal pumping rate determined through numerical analysis conducted by Aphale et al [24]. Indeed, Aphale et al (2006) show that only when the rate of lubricant supply determines that the inlet pressure remains below the outlet pressure air is entrained into the contact and the drag torque is reduced. In the case when $p_{i}-p_{o}>0$ the outer radius of the oil film region is the external radius of the disc (i.e. $R_{o}=R_{e}$ ). The pressure differential between the inlet and outlet is given in table 2 . This value is typical of the magnitude given by surface tension $\frac{2 \sigma \cos \theta}{h}$ [25] between the two plates. 
Now that the location of the free boundary $R_{o}$ is determined, the frictional drag and power loss acting on the wetted area of the brake disc can be evaluated. This section of the model only considers the flow in the tangential direction. As shown in figure 3 , the brake disc is made of two regions of discrete separation, the smaller of the two is the distance between the top of the friction pads and the stator; $h_{p}$. The second region is the distance between the stator and bottom of the grooves. The number of grooves $(n)$ and the groove width $(w)$ are also included to determine the area fraction with radius.

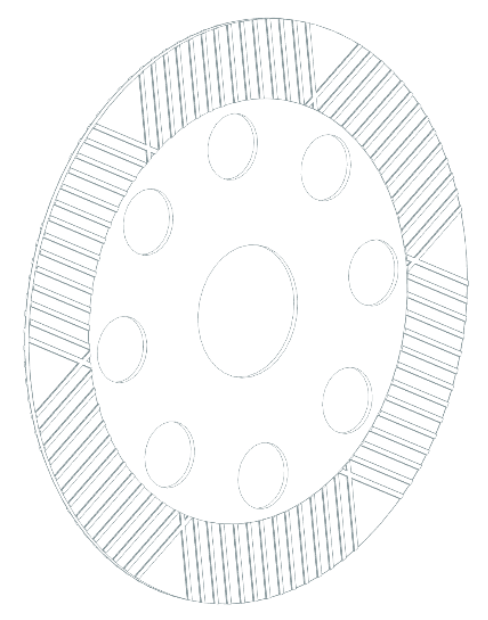

Figure 3: Brake disc geometry

Assuming that any Poiseuille flow in the tangential direction, caused by radial groove geometry is negligible, the Couette flow shear dominates, thus:

$T=\int_{R_{i}}^{R_{o}} \frac{\omega \eta r^{2}}{h_{p}}(2 \pi r-n w)+\frac{\omega \eta r^{2}}{h_{g}}(n w) d r$

$T=\omega \eta\left(\frac{\pi}{2 h_{p}}\left(R_{o}^{4}-R_{i}^{4}\right)+\frac{n w\left(R_{o}^{3}-R_{i}^{3}\right)\left(h_{p}-h_{g}\right)}{3 h_{p} h_{g}}\right)$

In the case of radial grooves, the term ( $\mathrm{nw}$ ) in equation (13) is independent of the radius. However, this term can simply be replaced for different groove patterns with a function of groove area with radius. Finally, the interfacial power loss can be found as:

$P=T \omega$

When disengaged, the frictional power loss in the contact causes shear heating of the lubricant film. To determine the primary mechanism through which heat is transferred the dimensionless thin film Peclet number for radial flow is used to determine the ratio of convective heat by the lubricant to the conduction through the bounding surfaces $[26,27]$.

$P e=\frac{2 c_{p} \rho Q}{\pi \lambda R_{i}}$

The Peclet number shows that for conditions during which there is likely to be significant shear heating (i.e. high parasitic losses) convective heat transfer is the most significant mechanism through which heat can be dissipated. First order approximation of mean temperature within the contact can be otained as [28]: 
$\theta=\theta_{0}+\frac{P}{2 c_{p} \rho Q}$

Where $\theta_{0}$ is the bulk sump temperature, $c_{p}$ is the specific heat capacity of oil and $Q$ is the oil flow rate through the conjunction. The flow rate is conserved throughout, the most convenient point to determine the flow rate is at the outer radius of the lubricated oil film extent. Using the integral in equation (18), the flow rate can be calculated through integration of $u$ at $r=R_{o}$ :

$Q=\frac{\pi R_{o}^{2} \rho \omega^{2} h_{p}^{3}}{45 \eta}+\frac{\mathrm{n} \mathrm{w} R_{o} \rho \omega^{2}\left(h_{g}^{3}-h_{p}^{3}\right)}{90 \eta}$

The influence of temperature change on lubricant's effective viscosity can be calculated using the Reynolds viscosity-temperature relationship:

$\eta=\eta_{0} e^{-\beta(\Delta \theta)}$

Where the temperature viscosity coefficient $(\beta)$ is given in table 1 . The system of equations $(14,15,17$, 18 and 19) can now be solved iteratively to determine the drag torque and power loss, including the influence of shear heating.

\section{Results}

The data in tables 1 and 2, representative of a typical range of vehicle operating conditions are used in the current analysis.

Table 1: Lubricant parameters

\begin{tabular}{lcc}
\hline Parameter & Value & Unit \\
\hline Dynamic Viscosity at $45^{\circ} \mathrm{C}\left(\eta_{0}\right)$ & 0.095 & $\mathrm{~Pa} . \mathrm{S}$ \\
Temperature viscosity coefficient $(\beta)$ & 0.287 & - \\
Density $(\rho)$ & 880 & $\mathrm{~kg} / \mathrm{m}^{3}$ \\
Specific heat capacity $\left(c_{p}\right)$ & 2200 & $\mathrm{~J} / \mathrm{kg} \cdot \mathrm{K}$ \\
Thermal conductivity $(\lambda)$ & 0.1 & $\mathrm{~W} / \mathrm{m} . \mathrm{K}$ \\
\hline
\end{tabular}

Table 2: Plate geometric parameters

\begin{tabular}{lcc}
\hline Parameter & Value & Unit \\
\hline Inner Radius $\left(R_{i}\right)$ & 0.08 & $\mathrm{~m}$ \\
External Radius $\left(R_{e}\right)$ & 0.11 & $\mathrm{~m}$ \\
Groove number $(n)$ & 84 & - \\
Groove width $(w)$ & 1.8 & $\mathrm{~mm}$ \\
Pressure differential $\left(p_{o}-p_{i}\right)$ & 450 & $\mathrm{~Pa}$ \\
Minimum separation $\left(h_{p}\right)$ & 200 & $\mu \mathrm{m}$ \\
Groove depth $\left(h_{g}\right)$ & 500 & $\mu \mathrm{m}$ \\
\hline
\end{tabular}

The developed model is validated against experimental measurements collected from the component level test rig at various temperatures and operating angular velocities. These are the two key parameters influencing the disengaged brake losses during operation. The lower angular velocity range $(0-350 \mathrm{rpm})$ is representative of the axle speeds typical of vehicle re-handling operations. The 
upper range of angular velocities (500-1000 rpm) represent typical axle angular velocities found when the vehicle is at maximum speed during on-highway travel. The temperature range investigated (45$100^{\circ} \mathrm{C}$ ) encapsulates the bulk oil sump temperatures found in application under various drive cycles and atmospheric conditions.

The results presented in the following section are for a single brake disc rotating between two stator plates (two interfaces). It should be noted the model presented in the previous section is for the calculation of a single brake disc stator interface. The model results have been multiplied by a factor of two to reflect the number of disc stator interfaces present in the experimental test rig.

Figure 4 shows close conformity of the analytical predictions with experimental measurements, including for oil film separation across a range of representative speeds and temperatures. The model is shown to accurately predict the power loss variation with angular velocity. At low speed conditions (below $350 \mathrm{rpm}$ ), the drag power loss varies with the square of the angular velocity. As the centrifugal force generated by the lubricant film increases, separation occurs, reducing the region over which a fluid film is subject to shear. After this critical angular velocity the power loss in the conjunction stabilises as the radius of the free boundary reduces with increasing speed. The angular velocity at which this occurs is predicted well by the developed model, as it appears in the subsequent reduction in area of the sheared lubricant film after this point. The second dashed line indicates the power loss predicted if the lubricant film does not rupture. In all cases, the model without oil film rupture closely agrees with the experimental data for the lower speed range. In the higher angular velocity range the model without rupture $\left(R_{o}=R_{e}\right)$ continues to indicate increasing losses, whilst the experimental and separation models predict stabilisation of power loss.

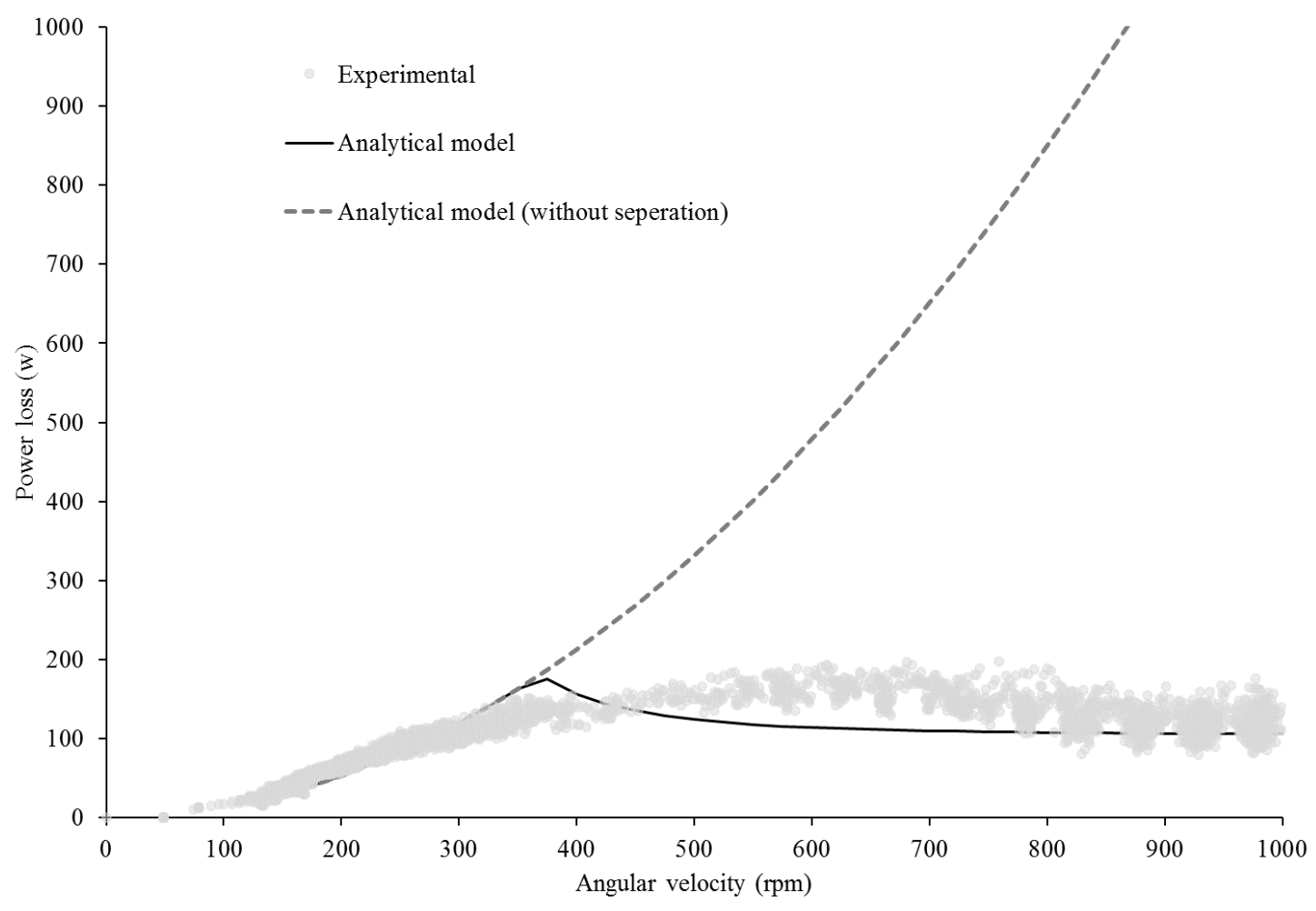

(a) 


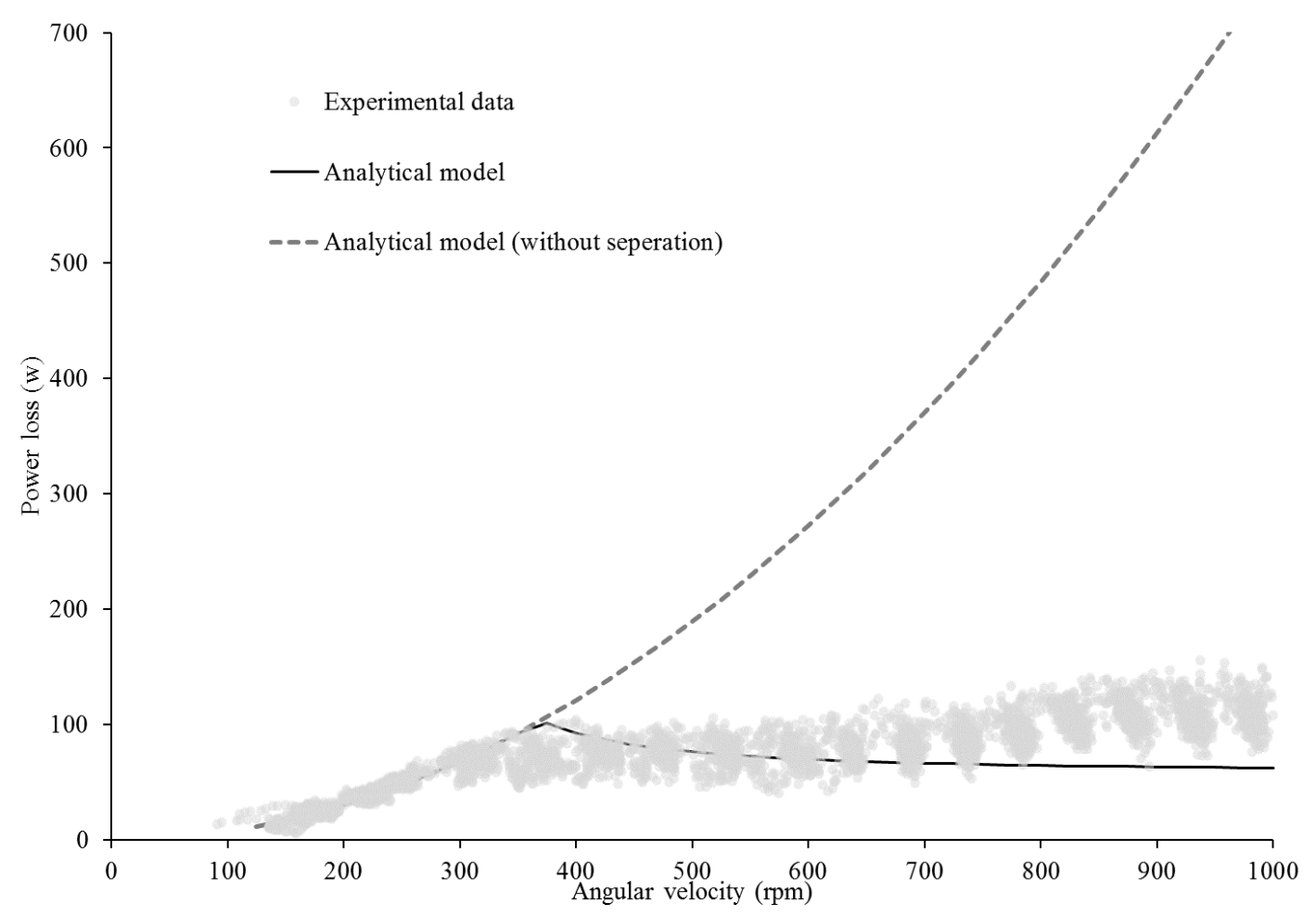

(b)

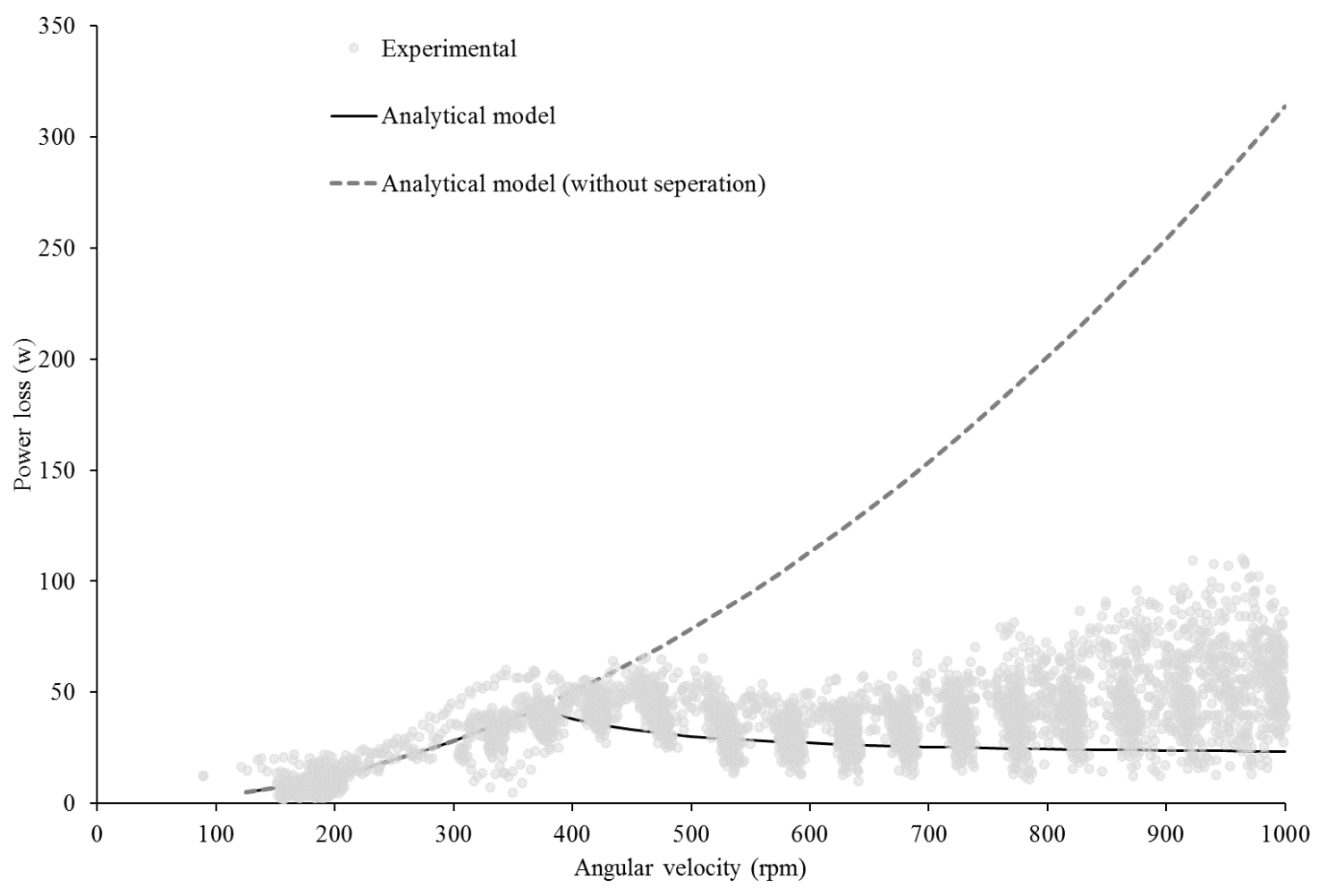

(c) 
Figure 4: (a) $45^{\circ} \mathrm{C}$ (b) $65^{\circ} \mathrm{C}$ (c) $100^{\circ} \mathrm{C}$.Validation of the model prediction for power loss at various angular velocities using experimental results

The parasitic frictional power losses, in figure 4, exhibit a similar trend for the drag torque shown in figure 5. The drag torque in the initial region, where a coherent film of lubricant is present between the two plates, varies in a linear fashion with angular velocity. Again, a critical effect is observed when the centrifugal forces and pressure differential between the boundaries create a reverse flow region and cause lubricant film separation. After this point any increase in shear of the fluid film is counteracted by reduction in outer radius of the fluid film region. The analytical model without film rupture is shown with a dashed line. It can be clearly seen without the separation model significantly over-predicts with respect to the experimental measurements. However, the separation model slightly under-predicts the experimental drag torque in the higher speed region once separation has occurred. This is likely to be due to the ingression of lubricant into the separated region in the submerged section of the disc. This effect is not accounted for in the model. It must also be noted that a thorough analysis of the rheological properties of the aerated oil is beyond the current analysis.

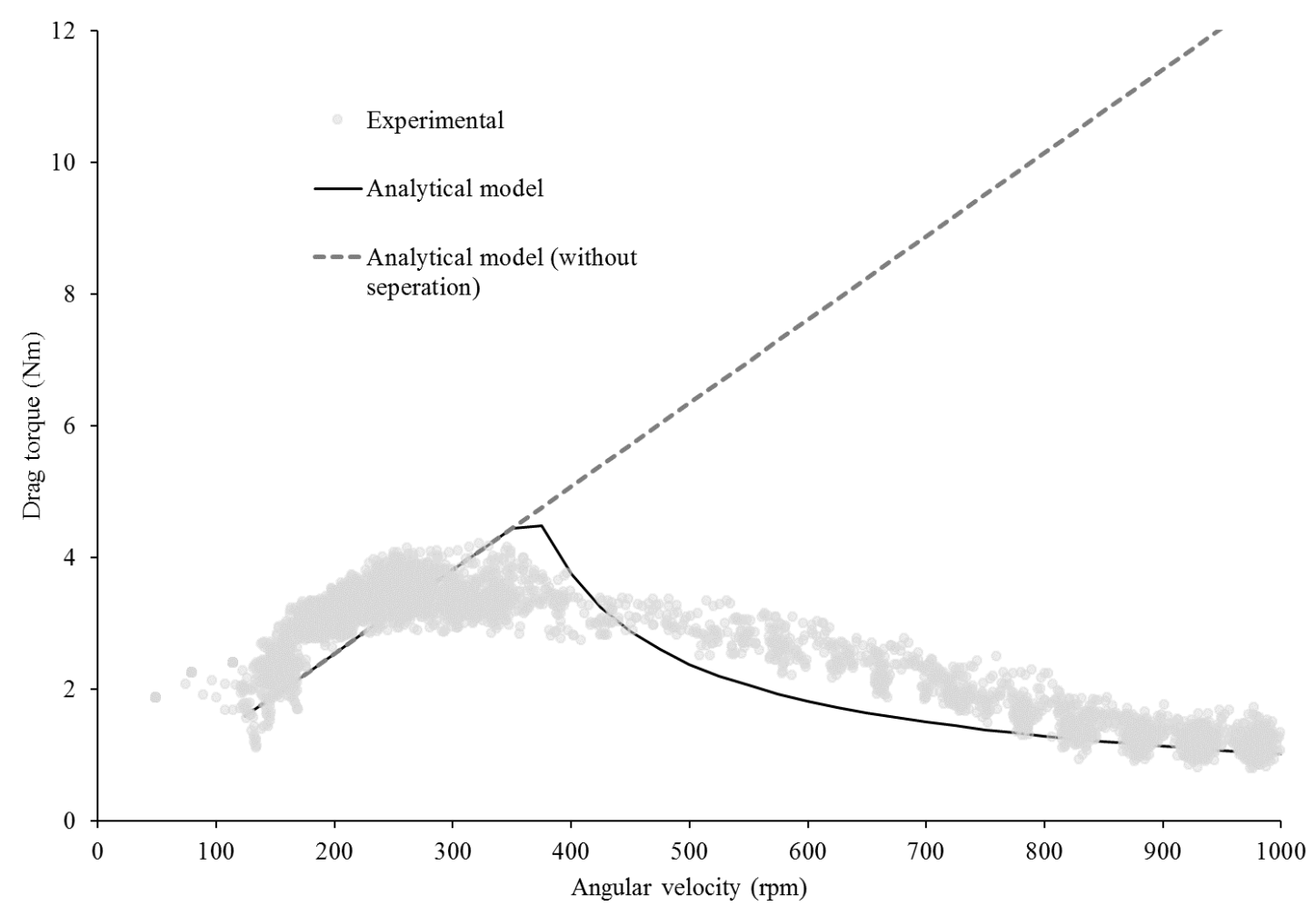

(a) 


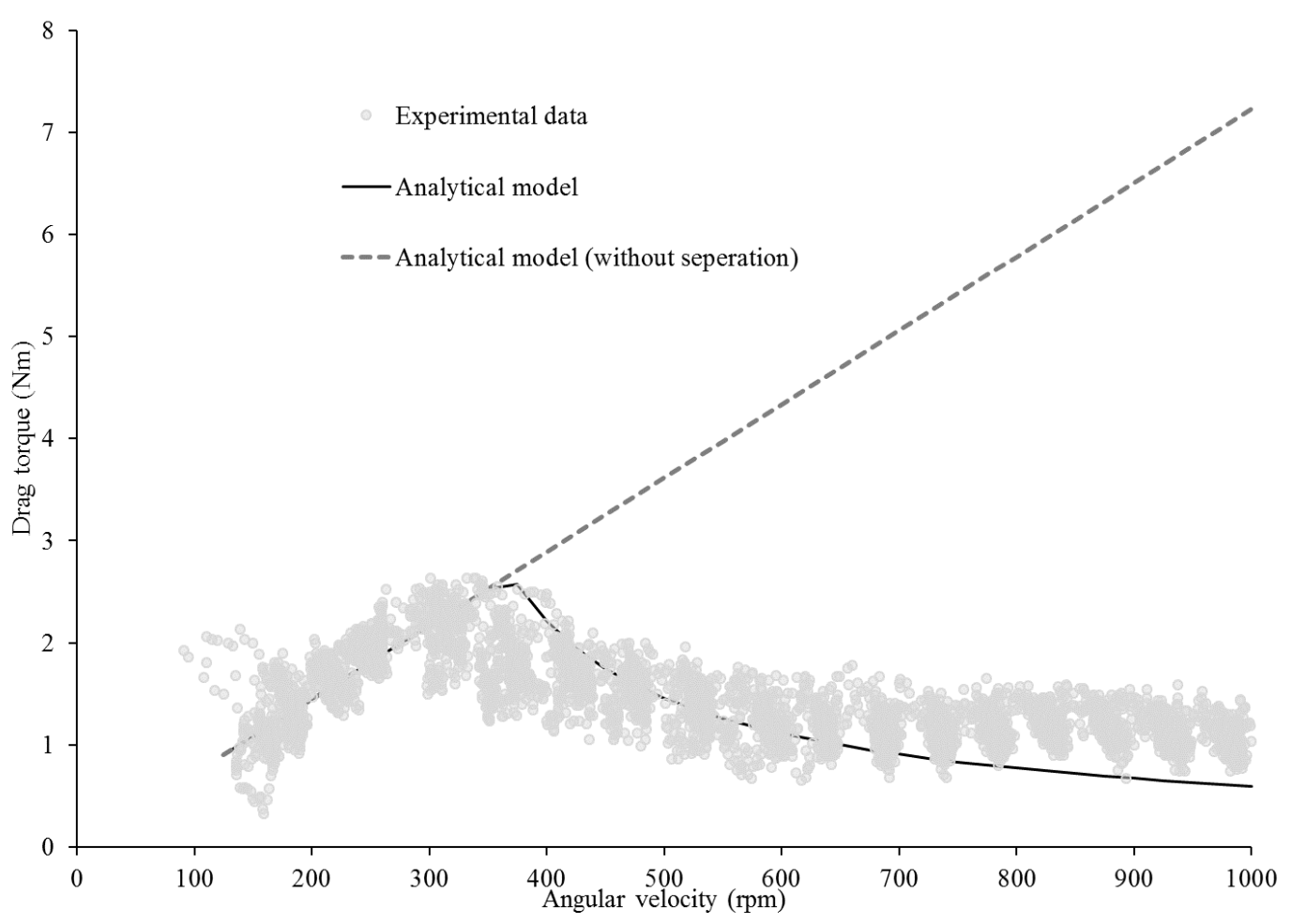

(b)

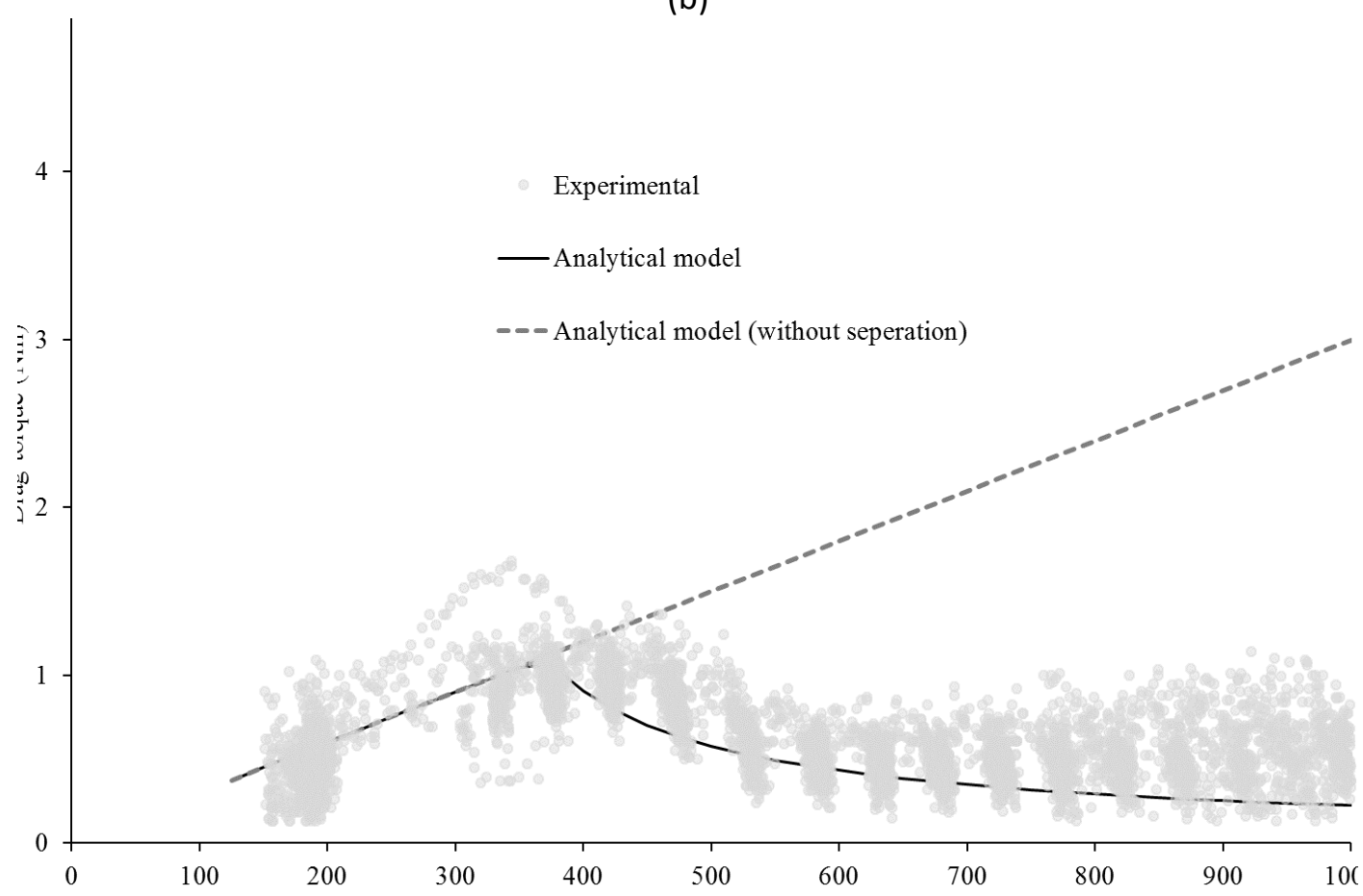

(c)

Figure 5: Validation of the model prediction of Drag torque at various angular velocities using experimental results(a) $45^{\circ} \mathrm{C}$ (b) $65^{\circ} \mathrm{C}$ (c) $100^{\circ} \mathrm{C}$.

The change in radius of the oil separation position is shown in figure 6 . The reduced outer radius can be seen to commence at the angular speed of $350 \mathrm{rpm}$. The two dashed lines represent the geometric inner and external radii of the brake disc. With the subsequent rise in the angular velocity the film 
separation position and outer radius of the oil film move towards the inner disc radius, reducing the wetted area subject to shear.

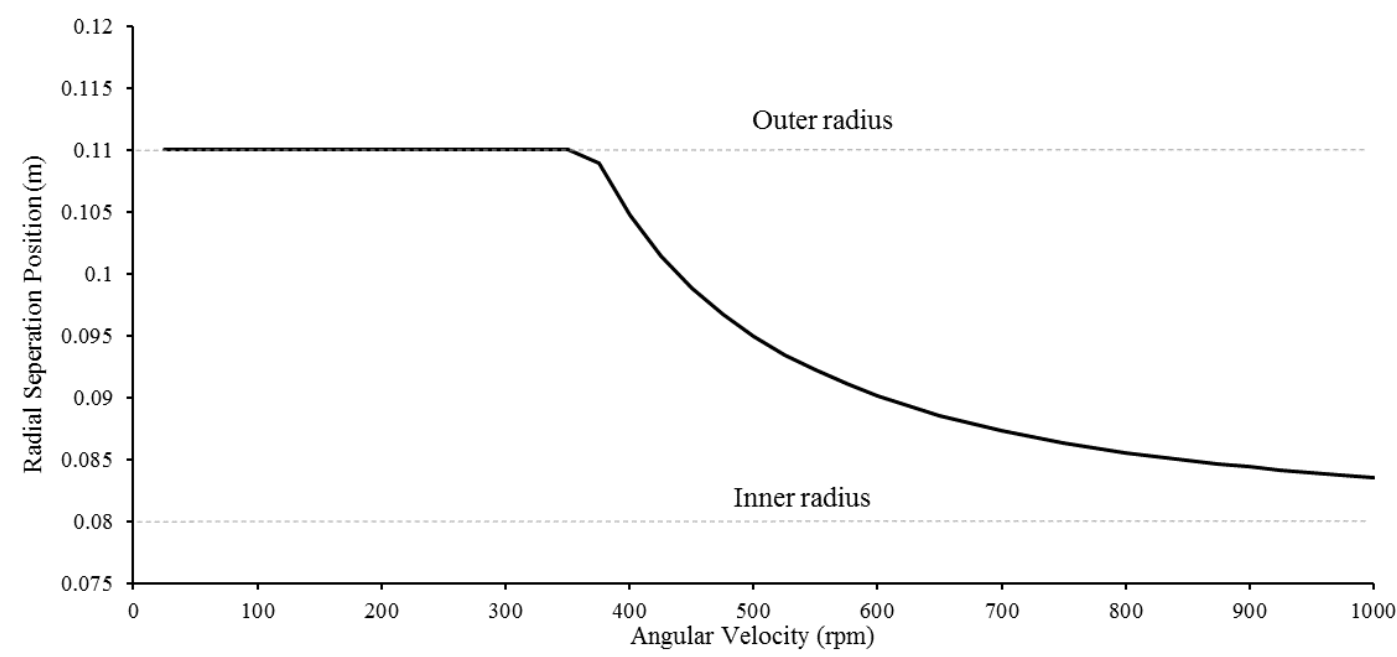

Figure 6: Variation of radial position of Oil film separation position at varying angular velocities

\section{Conclusions}

A novel model is presented to predict the disengaged drag losses in wet brake packs. The model uses the incipient flow reversal to determine the free boundary. For the current configuration the pressure gradient at which separation occurs is determined to be $\frac{d p}{d r}=\frac{r \rho \omega^{2}}{6}$. The method shown in the paper provides improved prediction when the mechanism of brake or clutch pack lubricant supply is through partial emersion or if the supply flow rate is unknown. The oil film separation model is shown to be in close agreement with the observed behaviour and in excellent agreement with experimental measurements.

The model predictions and experimental measurements highlight the magnitude of disengaged wet brake losses at a variety of typical axle angular velocities and operating conditions. The model can be an important tool for component design through integrated driveline efficiency models.

\section{Acknowledgements}

This work was supported by the J.C. Bamford Excavators and Innovate UK.

\section{References}

[1]- Quick, D.C. and Sippel, L.F., "Design and development of wet disc brakes for agricultural tractors", SAE Technical Paper, No. 730863, 1973.

[2]- Wilhem, J.P. and Loomis, A.V., "Brake friction materials: A market survey", NASA Technical reports, 1975.

[3]- Jenane, C., Bashford, L.L. and Monroe, G., "Reduction of fuel consumption through improved tractive performance", J. Agricultural Eng. Res., 1996, 64(2), pp.131-138. 
[4]- Dolatabadi, N., Rahmani, R., Theodossiades, S., Rahnejat, H., Blundell, G. and Bernard, G., "Tribodynamics of a New De-Clutch Mechanism Aimed for Engine Downsizing in Off-Road Heavy-Duty Vehicles", SAE Technical Paper, No. 2017-01-1835, 2017.

[5]- Dolatabadi, N., Rahmani, R., Theodossiades, S., Rahnejat, H., Blundell, G. and Bernard, G., "Tribodynamics of hydraulic actuated clutch system for engine-downsizing in heavy duty off-highway vehicles", Proc. IMechE, Part D: J. Automobile Engineering, 2018, 0954407018756789.

[6]- Leighton, M., Morris, N., Trimmer, G., King, P.D. and Rahnejat, H., 2018. "Efficiency of disengaged wet brake packs", Proc. IMechE, Part D: J. Automobile Engineering, 2018, 0954407018758567.

[7]- Lloyd, F.A., "Parameters contributing to power loss in disengaged wet clutches", SAE Technical Paper, No. 740676, 1974.

[8]- Jibin, H., Zengxiong, P. and Chao, W., "Experimental research on drag torque for single-plate wet clutch", Trans. ASME, J. Tribology, 2012, 134(1): 014502.

[9]- Pahlovy, S.A., Mahmud, S.F., Kubota, M., Ogawa, M. and Takakura, N., "New Development of a Gas Cavitation Model for Evaluation of Drag Torque Characteristics in Disengaged Wet Clutches", SAE J. Engines, 2016, 9(2016-01-1137), pp.1910-1915.

[10]- Sun, D.C. and Brewe, D.E., 1992. "Two reference time scales for studying the dynamic cavitation of liquid films", Trans. ASME, J. Tribology, 1992, 114(3), pp.612-615.

[11]- Swift, H.W., "The stability of lubricating films in journal bearings", J. Instn. Civil Engineers, 1932, 233(1): 267.

[12]- Stieber, W., "Dus Schwimmlager”, Verein Deutscher, Berlin, Ingenieurre, 1933.

[13]- Zweig, J.E. and Sneck, H.J., "Two-fluid flow between rotating annular disks", Trans. ASME, J. Lubrication Tech., 1976, 98(2), pp.214-222.

[14]- Yuan, S., Guo, K., Hu, J. and Peng, Z., 2010. "Study on aeration for disengaged wet clutches using a two-phase flow model", J. Fluids Engineering, 2010, 132(11): 111304.

[15]- Sneck, H.J., "The Effects of Geometry and Inertia on Face Seal Performance-Laminar Flow", Trans. ASME, J. Lubrication Tech., 1968. 90(2), pp.333-341.

[16]-Coombs, J.A. and Dowson, D., "Paper 12: An Experimental Investigation of the Effects of Lubricant Inertia in a Hydrostatic Thrust Bearing" In Proc. IMechE Conf., 1964,179(10), pp. 96-114).

[17]- Tipei, N., "Boundary conditions of a viscous flow between surfaces with rolling and sliding motion", Trans. ASME, J. Lubrication Tech., 1968, 90(1), pp. 254-261.

[18]- Mohammadpour, M., Johns-Rahnejat, P.M., Rahnejat, H. and Gohar, R., "Boundary conditions for elastohydrodynamics of circular point contacts", Tribology Letters, 2014, 53(1), pp. 107-118.

[19]- Shahmohamadi, H., Mohammadpour, M., Rahmani, R., Rahnejat, H., Garner, C.P. and HowellSmith, S., "On the boundary conditions in multi-phase flow through the piston ring-cylinder liner conjunction", Tribology International, 2015, 90, pp. 164-174. 
[20]- Birkhoff, G. and Hays, D.F., "Free boundaries in partial lubrication", Studies in Applied Mathematics, 1963, 42(1-4), pp.126-138.

[21]- Dowson, D. and Taylor, C.M., "Cavitation in bearings", Annual Review of Fluid Mechanics, 1979, 11(1), pp.35-65.

[22]- Tang, T., Morris, N., Coupland, J. and Arevalo, L., "Cavitation bubble measurement in tribological contacts using digital holographic microscopy", Tribology Letters, 2015, 58(1), p.5.

[23]- Dowson, D., "Inertia effects in hydrostatic thrust bearings", Trans. ASME, J. Basic Engineering, 1961, 83(2), pp.227-234.

[24]- Aphale, C.R., Cho, J., Schultz, W.W., Ceccio, S.L., Yoshioka, T. and Hiraki, H., "Modeling and parametric study of torque in open clutch plates", Trans. ASME, J. Tribology, 2006, 128(2), pp.422430.

[25] - Yuan, Y., Liu, E.A., Hill, J. and Zou, Q., "An improved hydrodynamic model for open wet transmission clutches", J. Fluids Eng., 2007, 129(3), pp.333-337.

[26] - Brunetière, N., Tournerie, B. and Frene, J., "TEHD lubrication of mechanical face seals in stable tracking mode: Part 2-Parametric study", J. Tribology, 2003, 125(3), pp. 617-627.

[27] - Roy, G., Nguyen, C.T. and Lajoie, P.R., "Numerical investigation of laminar flow and heat transfer in a radial flow cooling system with the use of nanofluids", Superlattices and Microstructures, 2004, 35(3-6), pp.497-511.

[28] - Neupert, T., Benke, E. and Bartel, D., "Parameter study on the influence of a radial groove design on the drag torque of wet clutch discs in comparison with analytical models", Tribology Int., 2018, 119, pp.809-821. 